\title{
Nanorobots a future Device for Diagnosis and Treatment
}

\author{
Sarath kumar $\mathrm{S}^{1^{*}}$, Beena P Nasim², Elessy Abraham ${ }^{3}$
}

Department of Pharmaceutics, Nazareth College of Pharmacy

\begin{abstract}
:
This article focusses on the application of the nanorobots in diagnosis and treatment of some of the diseases like cancer, heart diseases, diabetes, gout etc. Nanorobots are controllable machines at the nanometer or molecular scale that are composed of nano scale components. With the modern scientific capabilities, it has become possible to attempt the creation of nanorobotic devices and interface them with the macro world for control. These devices are also a helpful tool in the drug delivery, which is a very important aspect of medical treatment. Nanorobots are capable of performing task like actuating, sensing, signalling, information processing and intelligence at the nanoscale. The most recent application of these devices are, the brain targeted delivery of drugs, glucose monitoring in diabetes patients, bone reconstruction, cancer treatment, removal of blood clots, nerve regeneration and protein and peptide drug delivery system. Thus they play a vital role in the field of biomedicine especially in the treatment of cancer, cerebral aneurysm, kidney stones removal and some other treatments that have greatest aid to save human lives. This review guides to the recent research on nanorobots in the bio medical applications.
\end{abstract}

Key words: Nanorobots; Nanotechnology; Pharmacyte; Respirocyte; Microbivores.

\section{Introduction}

The word "nano" originates from the Greek word "dwarf". The concept of nanotechnology was first elaborated in 1959 by Richard Feynman, a Nobel Prize winning physicist, in a lecture titled, "There's plenty of room at the bottom". He ended the lecture concluding "this is a development which I think cannot be avoided ${ }^{[1]}$ ". Since then, nanotechnology has found use in a myriad of applications including dental diagnosis, material and therapeutics ${ }^{[1]}$. The term nanotechnology was coined by a student at a Tokyo science university in $1974^{[2]}$.

Nanotechnology is the study, design, creation, synthesis, manipulation, and application of materials, devices, and systems at the nanometer scale (One meter consists of 1 billion nanometers). Nanotechnology can best be defined as a description of activities at the level of atoms and molecules that have applications in the real world. A nanometre is a billionth of a meter, that is, about $1 / 80,000$ of the diameter of a human hair, or 10 times the diameter of a hydrogen atom ${ }^{[3-5]}$. Nanotechnology is a part of applied science whose theme is to control the matter on atomic and molecular scale ${ }^{[6]}$. It is becoming increasingly important in fields like engineering, agriculture, construction, microelectronics and health care to mention a few. The application of nanotechnology in the field of health care has come under great attention in recent times. There are many treatments today that take a lot of time and are also very expensive. Using nanotechnology, quicker and much cheaper treatments can be developed ${ }^{[7,8]}$.

Nanorobots: Nanorobots are the nanodevices that are used for protecting or treatment against pathogens in humans. It is a tiny device which is designed to perform a particular task or sometimes tasks with precision at nanoscale dimensions of 1-100 nm. They are expected to work at atomic, molecular and cellular levels to perform tasks in both medical and industrial fields $\mathrm{s}^{[6]}$. According to nanorobotic theory, "nanorobots are microscopic in size, it would probably be necessary for very large numbers of them to work together to perform microscopic and macroscopic tasks" ${ }^{[1]}$. Advances in the areas of robotics, nano structuring,
Received date: February 3, 2018

Accepted date: July 28, 2018

Publish date: August 5, 2018

*Corresponding author: Sarath kumar S. Department of Pharmaceutics, Nazareth College of Pharmacy, Email: sarathkumars1821995@gmail.com

Citation: Sarath, K.S., et al. Nanorobots a future Device for Diagnosis and Treatment (2018) J Pharm Pharmaceutics 5(1): 44-49.

Copyright: (C) 2018 Sarath, K.S. This is an Open access article distributed under the terms of Creative Commons Attribution 4.0 International License. 
medicine, bioinformatics, and computers can lead to the development of nanorobot drug delivery system. Some of the examples of Nanorobots are respirocytenanorobots, microbivoresnanorobots, surgical nanorobots and cellular repair Nanorobots.

Nanorobots will be used for maintaining and protecting the human body against pathogens. They will have a diameter of about 0.5 to 3 microns and will be constructed out of parts with dimensions in the range of 1 to 100 nanometres. The main element used Nanorobots is carbon because of its inertness and strength in the form of diamond and fullerene. Nanorobots have exterior passive diamond coating especially to avoid attack by the host immune system ${ }^{[3]}$. They are invisible to our naked eye, which makes them hard to manipulate and work with. Techniques like Scanning Electron Microscopy (SEM) and Atomic Force Microscopy (AFM) are being employed to establish a visual and haptic interface to enable us to sense the molecular structure of these nano scaled devices. Virtual Reality (VR) techniques are currently being explored in nano-science and bio-technology research as a way to enhance the operator's perception by approaching more or less a state of 'full immersion' or 'telepresence'. Fabrication and controls of the nanorobots are the main challenges in the development of nanorobots or nano machine components. Such devices will operate in microenvironments whose physical properties differ from those encountered by conventional parts. Nanorobotics is a field which calls for collaborative efforts between physicists, chemists, biologists, computer scientists, engineers and other specialists to work towards this common objective. Currently this field is still evolving, but several substantial steps have been taken by great researchers all over the world and are contributing to this ever challenging and exciting field ${ }^{[7]}$.

\section{Ideal characteristics of nanorobots}

- Nanorobots must have size in between 0.5 to 3 microns large with 1-100 nm parts.

- Nanorobots of larger size than the above will block capillary flow.

- It will prevent itself, from being attacked by the immune system by having a passive, diamond exterior.

- It will communicate with the doctor by encoding messages to acoustic signals at carrier wave frequencies of 1-100 MHz.

- It might produce multiple copies of it to replace worn-out units, a process called self-replication ${ }^{[6]}$.

\section{Types of nanorobots}

Some researchers classify nanorobots in drug delivery and therapeutics according to the their applications, which are described below,

Pharmacyte: It is a medical nanorobot having a size of 1-2 $\mu \mathrm{m}$ able to carrying up $1 \mu \mathrm{m} 3$ a given drug in the tanks. They are controlled using mechanical systems for sorting pumps. They are provided with a molecular markers or chemotactic sensors that guarantee full targeting accuracy. Glucose and oxygen extracted from the local environments such as blood, intestinal fluid and cytosol are the on board power supply. After the nanorobot completing tasks they can be removed or recovered by centrifuge nanapheresis.

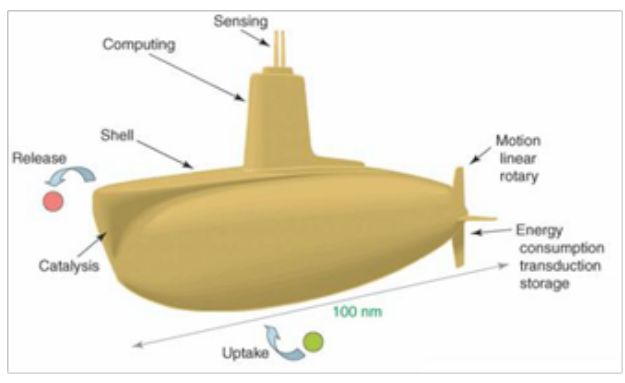

Figure 1: Fictitious Pharmacyte

Diagnosis and Imaging: They have microchips that are overlaid with human molecules. The chip is projected to send an electrical signal when the molecules detect a disease. Gives an example of special sensor nanobots that can be introduced into the blood under the skin where they verify blood contents and notify of any possible diseases. They can also be used to monitor the sugar level in the blood. Advantages are the low price to produce and easily to manipulate ${ }^{[9]}$.

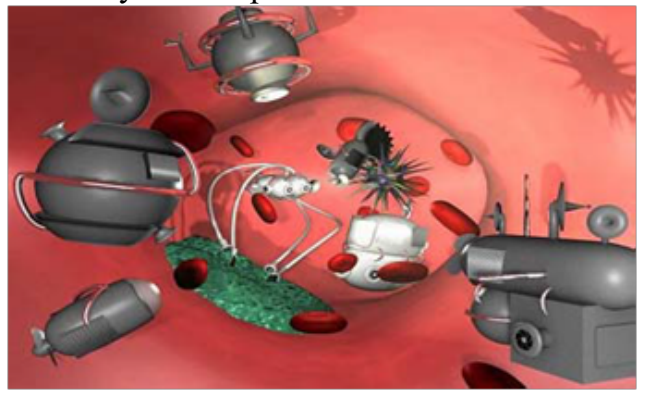

Figure: Nanorobots in blood vessel for Diagnosis and Imaging

Respirocyte: It is an Artificial Oxygen Carrier nanorobot which is about an artificial red blood cell. The power is obtained by endogenous serum glucose. This artificial cell is able to give 236 times more oxygen to the tissues per unit volume than RBCs (Red blood cells) and to administer acidity ${ }^{[10]}$.

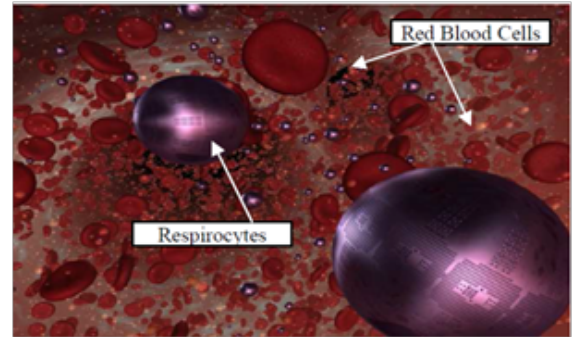

Figure: Respirocytes

Microbivores: It is an oblate spheroidal device for nanomedical applications with $3.4 \mu \mathrm{m}$ in diameter along its major axis and 2.0 $\mu \mathrm{m}$ in diameter along its minor axis. The nanobot can continually consume up to $200 \mathrm{pW}$. This power is used for digest trapped microbes. . Another distinctive feature is related to the ability to phagocyte approximately 80 times more efficiently than macrophages agents, in terms of volume/sec digested per unit volume of phagocytic agent. 


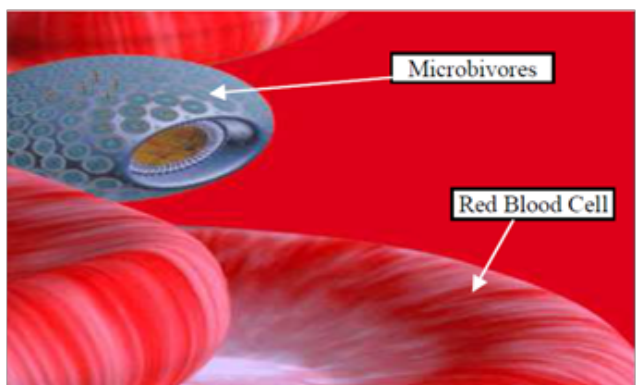

Figure: Microbivores

Clottocytes: This is a type of nanorobot, with a unique biological capability: "instant" hemostasis using clottocytes, or artificial mechanical platelets. It is known that platelets are roughly spheroidal nucleus-free blood cells measuring approximately 2 $\mu \mathrm{m}$ in diameter. Platelets join at a place of bleeding. There they are activated, becoming tacky and lumping together to form a tampon that aid stamp the blood vessel and stop the bleeding. They also delivery substances that help promote coagulating ${ }^{[10]}$.

Chromallocyte: The Chromallocyte would replace entire chromosomes in individual cells thus reversing the effects of genetic disease and other accumulated damage to our genes, preventing aging. Inside a cell, repair machine will first size up the situation by examining the cell's contents and activity, and then take action by working along molecule-by-molecule and structure-by structure; repair machines will be able to repair the whole cell.

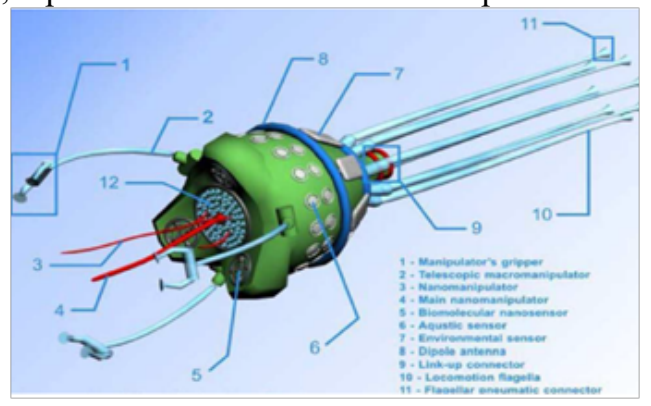

Figure: Diamondoid Cell-Repair Nanorobot

\section{Advantages of nanorobots}

- Use of nanorobot drug delivery systems with increased bioavailability;

- Targeted therapy such as only malignant cells treated;

- Reach remote areas in human anatomy not operatable at the surgeon's operating table;

- As drug molecules are carried by nanorobots and released where needed the advantages of large interfacial area during mass transfer can be realized;

- Non-invasive technique;

- Computer controlled operation with nobs to fine tune the amount, frequency, time of release;

- Better accuracy;

- Drug inactive in areas where therapy not needed minimizing undesired side effects ${ }^{[13]}$.

- Small size- The upper limit of the size of nanorobot is $3 \mathrm{mi}-$ cron so that it can easily flow in the body without blocking the capillary flow.

- Cost effective (if mass produced) - Manufacturing by batch processing reduces the cost even if the initial cost of develop- ment is high.

- Less post treatment care - As it is minimally invasive technique, therefore less post treatment care is required ${ }^{[11]}$.

Structure and design of nanorobots: The components of nanorobots are made of carbon because it is inert and possess good strength and in the form of diamonded or fullerene. The other components are hydrogen, oxygen, nitrogen, sulphur, silicon and fluorine, etc. which are used on nanoscale. The components of nanorobots are as follows;

Medicine cavity: It is a hollow section inside the nanorobot used to hold small doses of medicine in those robots which are capable of releasing medication directly to the site of injury or infection.

Probes, knives and chisels: These are used to remove plaque and blockages these probes, knives and chisels are used. These parts help Nanorobot to grab and break down the material. Also they might need a device to crush clots into very small pieces. If a partial clot breaks free and enters the bloodstream, it may cause more problems further down the circulatory system.

Microwave emitters and ultrasonic signal generators: These are used for destroying cells like cancerous cells without rupturing it. By using fine-tuned microwaves or ultrasonic signals, a nanorobot could break the chemical bonds in the cancerous cell, killing it without breaking the cell wall. Alternatively, the robot could emit microwaves or ultrasonic signals in order to heat the cancerous cell enough to destroy it.

Electrodes: With the help of electrodes nanorobots generate electric current, heating the cells until it dies.

Lasers: Lasers are used to burn the harmful materials like cancerous cells, blood clots and plaques i.e. these lasers vaporize tissues. With the help of powerful laser vaporizing cancerous cells is the challenging work, but this laser does not harm to surrounding tissues.

Power supply for nanorobots: The major requirement for the nanorobot is, of course, power. The nanorobots require power to allow it to perform all of its required operations. There are two possible ways.

The first is to obtain the power from a source within the body, either by having a self-contained power supply, or by getting power from the bloodstream.

The second possibility is to have power supplied from a source external to the body.

Medical application of nanorobots: Nanorobots are expected to enable new treatments for patients suffering from different diseases and will result in a remarkable advance in the history of medicine. The use of nanorobots may advance biomedical intervention with minimally invasive surgeries and help patients who need constant body functions monitoring, or ever improve treatments efficiency through early diagnosis of possible serious diseases. For example, the nanorobots may be utilized to attach on transmigrating inflammatory cells or white blood cells, thus 
reaching inflamed tissues faster to assist in their healing process. Some of the applications of nanorobots are as follows,

- Application of nano robots in dentistry

\section{Nanorobotic dentifrices (Dentifrobots):}

These when delivered either by mouthwash or tooth paste, can cover all sub gingival surfaces, thereby metabolizing trapped organic matter into harmless and odourless vapours. Properly configured dentifrobots can identify and destroy pathogenic bacteria that exist in the plaque and elsewhere. These invisibly small dentifrobots are purely mechanical devices that safely deactivate themselves when swallowed ${ }^{[1]}$.

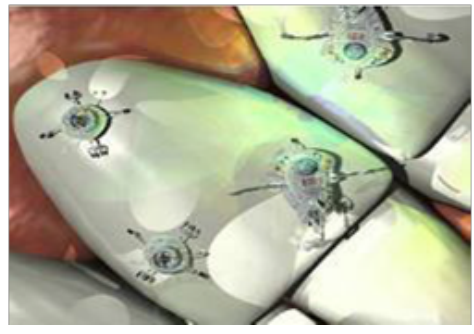

Figure: Dentifrobots

Nanoanesthesia: Oral anaesthesia is the most common procedures in dental practice, for that dental professionals will instil a colloidal suspension containing millions of active analgesic micron-sized dental nanorobot 'particles' on the patient's gingiva. After contacting the surface of the crown or mucosa, the ambulating nanorobots reach the dentin by migrating into the gingival sulcus and passing painlessly through the lamina propria or the 1 to 3-micron thick layer of loose tissue at the cement identinal junction. On reaching dentin, the nanorobots enter dentinal tubules holes that are 1 to 4 microns in diameter and proceed toward the pulp, guided by a combination of chemical gradients, temperature differentials, and even positional navigation, all under the control of the onboard nano computer as directed by the dentist. Tubules diameter increases as it nears the pulp, which may facilitate nanorobot movement. Once installed in the pulp and having established control over nerve impulse traffic, the analgesic dental nanorobots may be commanded by the dentist to shut down all sensitivity in any particular tooth that requires treatment. Then on the hand-held controller display, the selected tooth immediately becomes numb. After the oral procedures are completed, the dentist orders the nanorobots to restore all sensation, to relinquish control of nerve traffic and to engross, followed by aspiration. Nanorobotic analgesics offer greater patient comfort and reduced anxiety, no needles, greater selectivity, and controllability of the analgesic effect, fast and completely reversible switchable action and avoidance of most side effects and complications ${ }^{[15]}$.

Some other applications in the dentistry are tooth repair; Tooth repositioning, Dentin hypersensitivity etc.

Application of nano robots in cancer treatment: The patient will have more chances for treating from cancer if it diagnosed earlier. Nanorobots with chemical biosensor (nanosensor) are used for detecting the tumours cells in early stage of cancer development. This nanosensor will sense the presence of malignant cells in the body.

The therapeutic index of most anticancer drugs is narrow, causing toxicity to normal stem cells, hematological ad- verse effects, gastrointestinal among other. The Conventional chemotherapeutic agents work by destroying rapidly dividing cells, which is the main property of neoplastic cells. Most of the anti-cancer drugs like Doxorubicin are used in several types of cancer, such as HD (Hodgkin's disease), in which treatment is administered in combination with other antineoplastic agents in order to reduce their toxicity.

Considering the properties of nanorobots to navigate as blood borne devices, they can help on such extremely important aspects of cancer therapy. Nanorobots with embedded chemical biosensors can be used to perform detection of tumour cells in early stages of development inside the patient's body. Integrated nano sensors can be utilized for such a task in order to find intensity of Ecadherin signals. Therefore a hardware architecture based on nano bioelectronics is described for the application of nanorobots for cancer therapy ${ }^{[10]}$. The scientists have genetically modified salmonella bacteria that are drawn to tumors by chemicals secreted by cancers cells. The bacteria carry microscopic robots, about 3 micrometers in size that automatically release capsules filled with drugs when the bacteria reach the tumor. By delivering drugs directly to the tumor, the nanorobot, which the team named bacteriobot, attacks the tumor while leaving healthy cells alone, sparing the patient from the side effects of chemotherapy ${ }^{[6,9]}$.

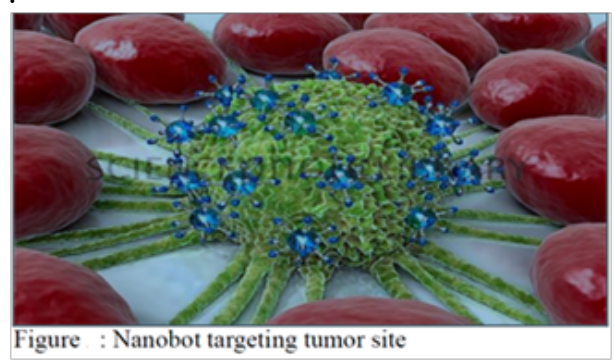

Figure: Nanorobots targeting tumor site

Nanorobots in the diagnosis and treatment of diabetes: Nanorobots are considered a new possibility for the health sector to improve medical instrumentation, diagnosis, and treatment of diabetes. Patients with diabetes must take small blood samples many times a day to control glucose levels. Such procedures are uncomfortable and extremely inconvenient. To avoid this kind of problem the level of sugar in the body can be observed via constant glucose monitoring using medical nanorobotics.

The simulated nanorobot prototype model has embedded Complementary Metal Oxide semi-conductor (CMOS) nanobioelectronics. It features a size of $\sim 2$ micronmeter, which permits it to operate freely inside the body. The nanorobot uses embedded chemosensor that involves the modulation of $\mathrm{hS}$ $\mathrm{GLT}_{3}$ protein glucosensor activity. Through its onboard chemical sensor, the nanorobot can thus effectively determine if the patient needs to inject insulin or take any further action, such as any medication clinically prescribed. They flow with the RBCs through the bloodstream detecting the glucose levels. At a typical glucose concentration, the nanorobots try to keep the glucose levels ranging around $130 \mathrm{mg} / \mathrm{dl}$ as a target for the Blood Glucose Levels (BGLs). In the medical nanorobot architecture, the significant measured data can be then transferred automatically through the RF signals to the mobile phone carried by the patient. At any time, if the glucose achieves critical levels, the nanorobot emits an alarm through the mobile phone. 


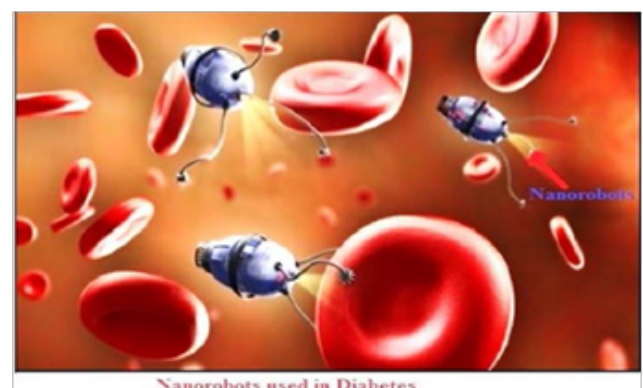

Figure: Nanorobots used in diabetes

An artificial oxygen carrier nanorobot: "Respirocyte" is the artificial mechanical red cell, an imaginary nanorobot which floats along in the blood stream. It is essentially a small pressure tank that can be pumped full of oxygen $\left(\mathrm{O}_{2}\right)$ and carbon dioxide $\left(\mathrm{CO}_{2}\right)$ molecules. Later on, these gases can be released from the small tank in a controlled manner. These atoms are mostly carbon atoms arranged as diamond in a porous lattice structure inside the spherical shell.

Outside of each device there are gas concentration sensors. When the nanorobot passes through the lung capillaries, $\mathrm{O}_{2}$ partial pressure is high and $\mathrm{CO}_{2}$ partial pressure is low, so the onboard computer tells the sorting rotors to load the tanks with oxygen and to dump the $\mathrm{CO}_{2}$. When $\mathrm{CO}_{2}$ partial pressure is relatively high and $\mathrm{O}_{2}$ partial pressure relatively low the onboard computer commands the sorting rotors to release $\mathrm{O}_{2}$ and to absorb $\mathrm{CO}_{2}$. Respirocytes simulate the action of the natural hemoglobin-filled red blood cells, but they can deliver 236 times more oxygen per unit volume than a natural red cell.

As artificial phagocyte (Microbivore): Microbivore is an artificial mechanical phagocyte of microscopic size whose primary function is to destroy microbiological pathogens found in the human bloodstream, using the "digest and discharge" protocol. The chief function of microbivore is to wipe out microbiological pathogens found in the human bloodstream, using the "digest and discharge" procedure. Microbivores upon given intravenously (I.V) would achieve complete clearance of the most severe septicemic infections in hours or less, far better than the weeks or months needed for antibiotic-assisted natural phagocytic defenses ${ }^{[6]}$.

Heart-attack prevention: Nanorobots can also be used to prevent heart-attacks. Heart-attacks are caused by fat deposits blocking the blood vessels. Nanorobots can be made for removing these fat deposits. The nanorobots remove the yellow fat deposits on the inner side of blood vessels. This will allow for both improving the flexibility of the walls of the arteries and improving the blood flow through them ${ }^{[18]}$.

From this hypothesis, such technology will help for delivery of drugs like lipid lowering substances such as lovastatin, simvastatin etc. These drug molecules will enter with nanorobots and give delivery at the site of action ${ }^{[2,8]}$.

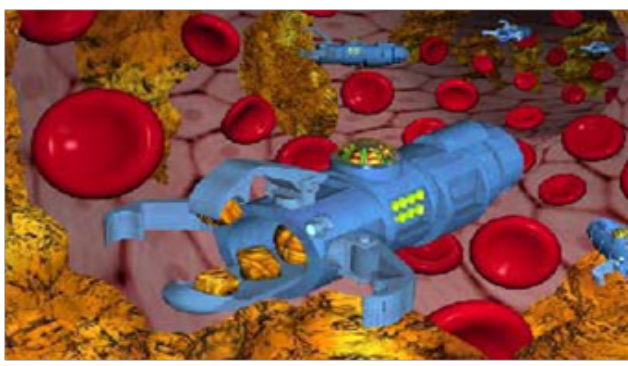

Figure: Nanorobots removing fat deposit

Cleaning wounds: Nanorobots could help remove debris from wounds, decreasing the likelihood of infection. They would be particularly useful in cases of puncture wounds, where it might be difficult to treat using more conventional methods ${ }^{[14]}$.

Nanorobots in kidney disease: Nanorobots are used to break the kidney stones with the help of ultrasonic shocks. Kidney stones are painful and a large stone does not pass out in urine. Sometimes doctor break this stones by ultrasonic frequency but, these are not effective in always. Nanorobots break up these kidney stones by using small laser and these smaller pieces are passing out in urine outside the body ${ }^{[2]}$.

\section{Conclusion}

Considering the severe side effects of the existing therapies like radiation and chemotherapy the nanorobots from the field of nanomedicine can be an innovative, supportive and optimistic machine technology for patients in the treatment and diagnosis of life threatening diseases. In future, the main emphasis in medicine will shift from medical science to medical engineering, where nanorobotic technology will be the revolution. Nanorobots applied to medicine hold a wealth of promise from eradicating disease to reversing the aging process (wrinkles, loss of bone mass and age-related conditions are all treatable at the cellular level); nanorobots are also candidates for industrial applications. They will provide personalised treatments with improved efficacy and reduced side effects that are not available today. They will provide combined action-drugs marketed with diagnostics, imaging agents acting as drugs, surgery with instant diagnostic feedback. This science might sound like a fiction now, but Nanorobotics has strong potential to revolutionize healthcare, to treat disease in future. It opens up new ways for vast, abundant research work. Future healthcare will make use of sensitive new diagnostics for an improved personal risk assessment. Highest impact can be expected if those major diseases are addressed first, which impose the highest burden on the aging population: cardiovascular diseases, cancer, musculoskeletal conditions, neurodegenerative and psychiatric diseases, diabetes, and viral infections ${ }^{[20,21]}$.

\section{References}

1. Mehra, P., Nabhi, K. A Nanorobotics. "The Changing Face of Dentistry“. (2016) (IJSR) 5(3): 192-197.

Pubmed| Crossref| Others

2. Nandkishor, K., Swapnil, P., Rajeshwar, K., et al. Review 
on application of nanorobots in health care. (2014) World J pharmacy and pharmaceutical sciences 3(5): 472-480. Pubmed| Crossref| Others

3. Sivasankar, M., Durairaj, R.B. Brief Review on Nano Robots in Bio Medical Applications. (2012) ARA 1(1): 101. Pubmed| Crossref| Others

4. Ummat, A., Dubey, A., Sharma, G., et al. Nanorobotics: $1-43$.

Pubmed| Crossref| Others

5. Kh.Hussan, R., Asiwarya. G., Radhika, .G., et al. Nanorobots: the future trend of drug delivery and therapeutics. (2011) 10(1): 60-68.

Pubmed| crossref| Others

6. Meena, K., Monika, N., Sheela, M., Nanorobots: A Future Medical Device in Diagnosis and Treatment. (2013) Research J Pharmaceutical, Biol Chemical Sci 4(2): 1229-1307 Pubmed| Crossref| Others

7. Debjit, B., Chiranjib, B., chandira, R.M., et al. Role of nanotechnology in novel drug delivery system. (2009) J Pharmaceutical Sci Technology 1 (1): 20-35.

Pubmed| crossref| Others

8. Sachin, S.S., Neela, M.B., Sachin S.M.,et al. Nanorobots: novel emerging technology in the development of pharmaceuticals for drug delivery applications. (2013) World J pharmacy and pharmaceutical sci 2(6): 4728-4744.

Pubmed| Crossref| Others

9. Rohit, K., Omprakash,B., Sanat, K., et al. Applications of Nanorobotics. (2014) International J of Scientific Res Eng \& Tech (IJSRET) 3(8): 1131-1136.

Pubmed| Crossref| Others

10.GléciaVirgolino, d.S., Kleber, V.G.B. Fábio Vladimir, C.d.A., et al. Nanorobotics in Drug Delivery Systems for Treatment of Cancer: A Review. (2016) J Mat Sci Eng A 6 (5-6) (2016): 167-180.

Pubmed| Crossref| Others

11. Jhansee, M., Alok,K.D., Rajeev, K. Nanotechnology Challenges; Nanomedicine: Nanorabots. (2012) Int. Res. J. of Pharmaceuticals 2(4): 112-119.

Pubmed| Crossref| Others

12. Abhilash, M. Nanorobots", International Journal of Pharma and Bio Sciences 1(1): 1-10.

Pubmed| Crossref| Others

13. Kal, R.S. Nanorobot Drug Delivery System for Curcumin for Enhanced Bioavailability during Treatment of Alzheimer's disease. (2013) J Encapsulation and Adso Sci 3(1): 24-34. Pubmed| Crossref| Others

14. Deepa, R., Parmar, J.P., Soni, A.D.,et al. Nanorobotics in Advances in Pharmaceutical Sciences. (2010) Int J of Drug
Dev \& Res 2(2): 247-256.

Pubmed| Crossref| Others

15. Maryam, M. Future of dentistry, nanodentistry, ozone therapy and tissue engineering. (2013) J Dev Biol and Tissue Eng 5(1): 1-6.

Pubmed| Crossref| Others

16. Ari, R., Bruce, k., Mark, T. Nanorobotics. (2003) NSF: 1618.

Pubmed| Crossref| Others

17. Prajapati, P.M., Solanki, A.S., Sen, D.J. Importance Nanorobotics in Health Care. (2012) Inter Res J Pharmacy 3(3): 122-124.

Pubmed| Crossref| Others

18. Adriano, C., Lior, R., Bijan, S., et al. Nanorobot for Treatment of Patients with Artery Occlusion. (2006) Proceedings of Virtual Concept 2006: 1-10.

Pubmed| Crossref| Others

19. Robert, A. F. Pharmacytes: An Ideal Vehicle for Targeted drug delivery. (2006) J of Nanosci and Nanotech 6(9-10): 2769-2775.

Pubmed| crossref| Others

20. Prabhjot, k., Loveleenpreet, kaur., Khan, M.U. Nanoparticles as a Novel Drug Delivery System: A Review. (2012) Int J Res Pharm and Chem 2(3): 756-761.

Pubmed| Crossref| Others

21. SakshiSethi, M. Nanorobotic Technology in the Medical Industry. (2015) Inte J Electr, Electrical and Compu Sys IJEECS 4(5): 46-50.

Pubmed| Crossref| Others

Submit your manuscript to Ommega Publishers and we will help you at every step:

- We accept pre-submission inquiries

- Our selector tool helps you to find the most relevant journal

- We provide round the clock customer support

- Convenient online submission

- Thorough peer review

- Inclusion in all major indexing services

- Maximum visibility for your research

Submit your manuscript at OMMEgA Publishers https://www.ommegaonline.org/submit-manuscript 